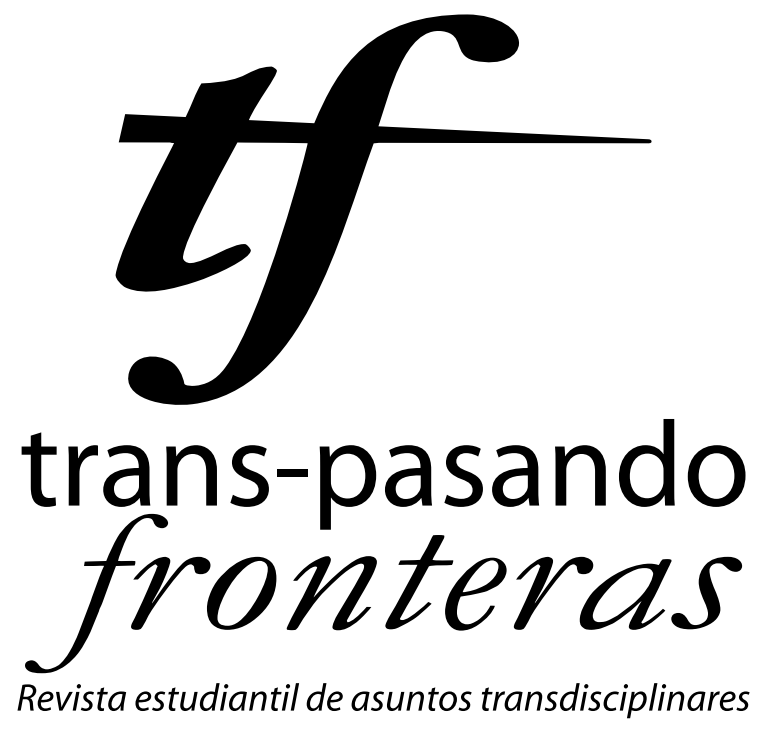

Una publicación de

Estudios

Interdisciplinarios

Jurídicos, Sociales

000 y Humanistas

ICESS

FACULTAD DE

DERECHO Y CIENCIAS

SOCIALES

然 UNIVERSIDAD 


\title{
Los Objetivos de Desarrollo del Milenio y la revolución de datos
}

\author{
Entrevista a Ivo Havinga \\ Jefe de la División de Estadística del \\ Departamento de Asuntos Económicos y Sociales (DAES) de \\ la Organización de las Naciones Unidas
}

\author{
Por Richard Benavidez \\ Estudiante de Ciencia Política con concentración en \\ Relaciones internacionales \\ (benavidez.richard@hotmail.com)
}

Richard Benavidez (RB): ¿Por qué es tan importante una Revolución de Datos para el Desarrollo Dostenible?

Ivo Havinga (IH): Para mí, la revolución de datos tiene que ver con la nueva transformación de cómo queremos ver el mundo para el 2015. Lo que deja en evidencia la necesidad de mayor transparencia en la rendición de cuentas. Por lo tanto, uno de los principales objetivos es poder proveer aquellos datos que son necesarios para informar a la comunidad con tal de que ellos puedan corroborar la información. Esto implica grandes desafíos en todo sentido, pero al final todos podremos hacer un mejor análisis de los resultados de las políticas que nos abordan.

Además de lo anterior, esto implica, necesariamente, un cambio en la manera en que percibimos los datos. La forma tradicional en la que los vemos es en las oficinas nacionales de estadística, estadísticas sobre la economía, fenómenos 


\section{"Debemos de trabajar verdaderamente juntos para tener acceso a la información sobre una base legal, así como deben de haber leyes en cada país que permita, a los estudiante y académicos, empezar a usar esta información"}

sociales y ambientales que informan a la ciudadanía y a los políticos sobre estos ámbitos.

Pienso que debemos ir más allá porque hay nuevas fuentes de datos disponibles que debemos de empezar a utilizar. Uno de las fuentes que se ha vuelto disponible son las imágenes satelitales, y las redes sociales; estos son nuevos escenarios de datos que se han vuelto importantes para nosotros. Para poder desarrollar esta fuentes debemos tener acceso a ese tipo de datos, normalmente estos datos son generados por empresas privadas, entonces debemos desarrollar asociaciones, y mantener la transparencia y calidad de los datos cuando la usamos.

También hay cuestiones tecnológicas que se deben abordar, por el volumen de los datos, el tipo de análisis que se debe de hacer es diferente. En este sentido tenemos asuntos legales respecto al acceso a los datos, asuntos de privacidad, porque los datos están básicamente relacionados 
con individuos. Entonces, todos estos son problemas que debemos abordar cuando se habla de una Revolución de Datos.

También se deben transformar esos datos en conocimiento porque los datos en sí no son suficientes, entonces debemos hablar de su transformación en conocimiento. $\mathrm{Y}$ eso, no es solamente un problema de la Oficina Nacional de Estadística (ONE).

RB: ¿Qué piensa acerca de la importancia de la calidad de los datos?

IH: Esto es muy importante, la mejor manera de usar los datos es encontrándoles un sentido, no sólo basarse en estándares de calidad. Tenemos estándares reconocidos a nivel internacional que nos ayudan a evaluar si la calidad de la información es buena o no. Pero también es trabajo de los analistas políticos, de la academia, y de los medios de comunicación, porque ellos los explican.

Cabe aclarar que no todos los medios de comunicación están bien equipados para escribir sobre algunos temas, entonces debemos ser capaces de educarlos en el uso de estos datos, y en la manera de informar a las personas. Las ONG tienen un rol muy importante: entender los problemas de comunidad. Los individuos (hombres, mujeres, niños o ancianos), también se deben comunicar sobre los problemas políticos porque los afectan. Es algo que llamamos "Asociación Global" y que se debe desarrollar. Todo esto hace parte de algo que llamamos La Revolución de Datos. Es algo muy grande, es la transformación de nuestra manera de ver las cosas.

RB: ¿Cuáles son los nuevos objetivos post-2015 para la Cooperación internacional?

IH: El problema es que nosotros a nivel internacional hemos aprendido mucho sobre establecer metas, objetivos e indicadores. Esto nace desde los Objetivos de Desarrollo del Milenio (ODM) desde la Cumbre del Milenio en el 2000 y después la cumbre de Johannesburgo en el 2002; donde básicamente decidieron la estructura de los ODM y fueron reconocidos como las metas que se querían lograr a nivel internacional. Por encima de todos los esfuerzos siempre está la reducción de la pobreza y el hambre extrema, pero también, de los problemas ambientales, sobre mujeres, niños, educación, salud, entre otros.

RB: ¿Cómo se encuentra Colombia en lo que respecta al medio ambiente?

IH: No tenemos un sistema de cla- 
sificación (ranking) sobre el medio ambiente. El DANE (Departamento Administrativo Nacional de Estadísticas) tiene información muy valiosa sobre el medio ambiente. Nosotros usamos esa información y trabajamos para mejorar los estándares e informar qué tipo de datos se debe presentar.

RB: ¿Cómo recoje las Naciones Unidas toda esa información?

IH: Déjame ponerlo de la siguiente manera: con los Objetivos del Milenio hubo un entendimiento del proceso de planeación de desarrollo de un país y con los Objetivos de Desarrollo Dostenible (ODS) hacemos exactamente lo mismo. Esa información debe ser parte de los principales indicadores de un país, y tienen que ver con el proceso de planificación que está relacionado con los ministerios de hacienda, planeación, medio ambiente. $\mathrm{Y}$ se busca que cada año se vayan mejorando los indicadores, aun si se ha cumplido el objetivo.

Lo que queremos hacer con todos estos indicadores y objetivos es incluirlos dentro de la planificación y programación general del país, a nivel público. Darle un sentido de orientación a la gestión pública en cada país. Somos parte del proceso, no solo obtenemos información de las oficinas nacionales; también de los bancos centrales brinda estadísticas económicas, pero también de los Ministerios del Medio Ambiente sobre el uso del bosque, el agua y otros recursos naturales como los océanos y la luz solar; ahora se obtienen datos de otras fuentes, aparte del sistema nacional de estadística.

RB: ¿Cómo se puede conectar la sociedad por medio de la creación de 'grandes datos' o Big Data?

IH: La Big Data, como lo he mencionado, son datos de gran volúmen que no vienen estandarizados. En el pasado, las fuentes tradicionales venían en un formato estándar; porque las usábamos, sabíamos qué tipo de información estaba recopilada para analizarla. La Big Data tiene un formato abierto, por lo que básicamente debemos desarrollar el área académica, a lo que llamamos "científicos de datos", que son capaces de aprender cómo sacar la información de esos grandes datos. Es ahí donde está la relevancia del papel de las instituciones educativas. Empezar a utilizar la Big Data en investigaciones de pregrado y de maestrías es importante para familiarizarse con las 
técnicas que se usan para analizarla. Este es un rol fundamental para la academia.

Y también para nosotros, porque al tener conocimiento de los grandes datos debemos explicar a otras personas que no saben de este tipo de información. Esto, es muy importante.

Del mismo modo, es importante para nosotros a nivel internacional, que a nivel regional los datos estén disponibles. Porque básicamente todo son "micro datos" que se deben anonimizar para evitar problemas de confidencialidad, que es otro aspecto importante. Debemos de trabajar verdaderamente juntos para tener acceso a la información sobre una base legal, así como deben de haber leyes en cada país que permita, a los estudiante y académicos, empezar a usar esta información.

RB: ¿Cuál es su perspectiva de la manera en la que la ONU investiga los datos?

IH: En la ONU nosotros básicamente usamos la información que sale sobre los ODM y los Objetivos de Desarrollo Sostenible (ODS) para reportar anualmente a la asamblea general el progreso y después basándonos en el progreso que vemos en los países nuevas políticas son desarrolladas. Como por ejemplo políticas de 'energía para todos', 'educación para todos', y todas estas iniciativas que están relacionadas a estos objetivos, para mejorar la vida de la comunidad y las personas.

En este momento vemos como teníamos un plan del 2000 al 2015 para alcanzar unas metas específicas, ahora tenemos iniciativas en los países que aceleran la realización de estos objetivos; entonces nosotros trabajamos con los países en el proceso de planeación para mejorar el bienestar de los ciudadanos. Del reporte anual, aparte de que aprendemos lecciones, tenemos países van a la ONU pidiendo asistencia para mejorar los resultados generales de los objetivos. Entonces de verdad está funcionando.

RB: Y después se plantearan nuevos objetivos...

IH: Exacto, porque se sigue adelante. Si la pobreza es erradicada, en algún punto se seguirá con otras áreas como la mejora del medio ambiente, siempre hay nuevas aspiraciones. Pero en estos momentos debemos concentrarnos en 'no más pobreza', 'no más hambre' en el mundo, y problemas relacionados. $\mathrm{Si}$ logramos superar esto y seguir adelante abran otras aspiraciones. Es decir, tuvimos los Objetivos de Desarrollo 
del Milenio del 2000 al 2015; después tendremos del 2015 a, probablemente, 2030 los ODS; y después está en sus manos decidir cuáles serán los próximos. Tienen que empezar a pensar en eso, es su responsabilidad, de su generación. Mi generación ahora está pensando en los ODS, ustedes pensaran en otros. Tal vez, ¿Cómo ir a la luna? ¿Cómo ir a Marte?

Ustedes tal vez lograrán una mejor integración de América Latina, tendrían los Estados Federados de América Latina. Sabes, puede haber las aspiraciones que quieran.

RB: Hay investigaciones sobre los nuevos problemas de América latina. Por ejemplo, los movimientos sociales un proceso que Colombia que acaba de empezar.

IH: Tal vez se refieren a problemas de inequidad. Por otro lado, pienso que hay muchas cosas para hacer en el espacio y los océanos, suena chistoso, pero dentro de 30 años habrá mucho más para hacer. Ustedes deben de pensar en eso. Es bastante responsabilidad, pero eso es lo que define su generación, esa es la belleza de pensar en la transformación de la sociedad. Uno de los asuntos principales es la rendición de cuentas y transpa- rencia de los estados y necesitamos los datos para informarnos. 